\title{
Anti-Roll Bar Modeling for NVH and Vehicle Dynamics Analyses
}

\author{
Jakub Tobolár $^{1} \quad$ Martin Leitner $^{1} \quad$ Andreas Heckmann $^{1}$ \\ ${ }^{1}$ German Aerospace Center (DLR), Institute of System Dynamics and Control, Wessling, \\ Jakub. TobolareDLR. de
}

\begin{abstract}
The latest extension of the DLR FlexibleBodies Library concerns the field of automotive applications, namely the anti-roll bar. For the particular purposes of NVH and vehicle dynamics, the anti-roll bar module provides two appropriate levels of detail, both being based upon the beam preprocessor. In this paper, the procedure on preparing the models and their application for particular automotive related analyses is presented.

Keywords: anti-roll bar, vehicle chassis, flexible body, beam model, finite element
\end{abstract}

\section{Introduction}

Whenever an automotive suspension is excited in vertical direction due to road irregularities or driving maneuvers in an asymmetrical way, i.e. differently on the right and the left side of the vehicle, the roll motion of the car body is stimulated. This concerns - in common case - the comfort and driving experience of the car passengers. In limit conditions' situations, such a roll motion can influence the road-holding forces in a way that vehicle's driving safety is affected significantly. Consequently, it is advantageous to introduce an additional suspension component in particular tailored to influence the dynamical roll motion characteristics independently from the layout of the vertical suspension. This so-called anti-roll bar (also called stabilizer or anti-sway bar), see e.g. (Rill, 2012) or (Heißing and Ersoy, 2011), connects the suspensions on the right and the left side of the vehicle's axle by a cranked bar that acts as a torsional spring, see Figure 1.

Therefore, the design of the anti-roll bar is mainly targeted on its torsional stiffness, but also has to comply with the available space at the underfloor and must allow for attachments to the vehicle body and to both vertical suspensions. These requirements quite often result in the anti-roll bar to be a geometrical complex structural element that is prone for dynamical vibrations.

In daily practice, the Finite Element (FE) method turned out to be the adequate tool to design the geometrical and the structural properties of anti-roll bars. However, the driving behavior of vehicles, to which the anti-roll bar significantly contributes, is commonly developed using multibody simulation - generally utilizing the MultiBody package of the Modelica Standard Library in the Modelica community. In addition, the DLR FlexibleBodies Library

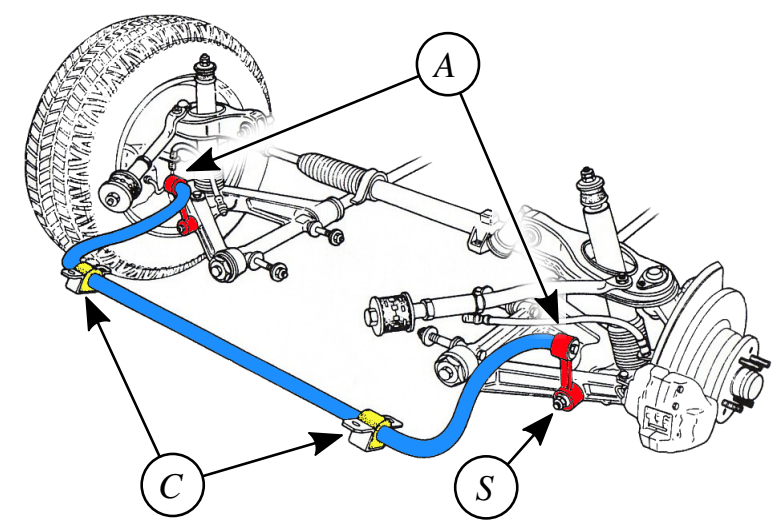

Figure 1. Vehicle axle with an anti-roll bar (color emphasized, courtesy of Wikimedia Commons).

(Heckmann et al., 2006) provides capabilities to incorporate data that originate from FE models in Modelica models. Thus, a tool chain to perform vehicle dynamics simulation including the structural characteristics of anti-roll bars is in principle available.

In common design tasks, driving maneuvers or noise, vibration and harshness (NVH) scenarios are first analyzed in multibody simulations. Then, the FE method is used to redesign the anti-roll bar in order to improve its characteristics. Subsequently, a FE to multibody interface has to be used to prepare the new FE data for the $D L R$ FlexibleBodies Library and, finally, the vehicle dynamics simulation has to be invoked again in order to assess the modification. This tool chain or loop, respectively, is inconvenient and makes it difficult to set up computational optimization procedures.

The given background motivates the introduction of a new modeling capability called AntiRollBar into the $D L R$ FlexibleBodies Library. In the present paper, a principle of the flexible body modeling and of the beam theory behind the AntiRollBar model is given in Sections 2.1 and 2.2, respectively. In Section 3, a framework of the AntiRollBar and its parametrization is discussed. Section 4 presents first simulation experiments provided.

\section{Theoretical Background}

\subsection{Flexible Bodies Theory}

The mechanical description of flexible bodies in multibody systems is based on the floating frame of reference 


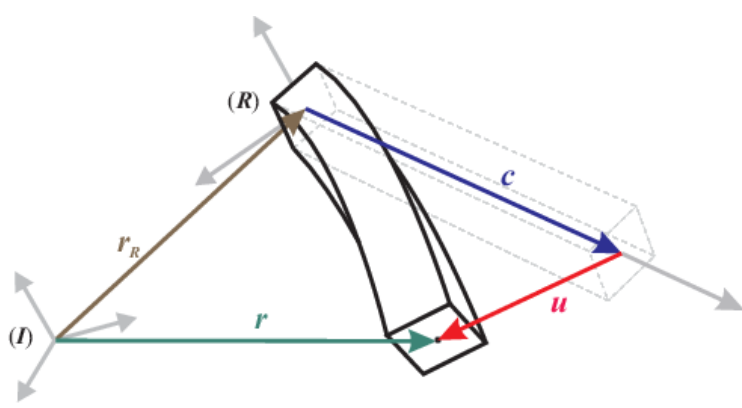

Figure 2. Vector chain of the floating frame of reference.

approach, i.e. the absolute position ${ }^{1} \boldsymbol{r}=\boldsymbol{r}(\boldsymbol{c}, t)$ of a specific body particle is subdivided into three parts:

- the position vector $\boldsymbol{r}_{R}=\boldsymbol{r}_{R}(t)$ to the body's reference frame,

- the initial position of the body particle within the body's reference frame, i.e. the Lagrange coordinate $\boldsymbol{c} \neq \boldsymbol{c}(t)$,

- and the elastic displacement $\boldsymbol{u}(\boldsymbol{c}, t)$ that is approximated by a Taylor expansion, here limited to first order terms, with space-dependent mode shapes $\boldsymbol{\Phi}(\boldsymbol{c}) \in \mathbb{R}^{3, n}$ and time-dependent modal amplitudes $\boldsymbol{q}(t) \in \mathbb{R}^{n}$, cf. (Wallrapp, 1994):

$$
\boldsymbol{r}=\boldsymbol{r}_{R}+\boldsymbol{c}+\boldsymbol{u}, \quad \boldsymbol{u}=\boldsymbol{\Phi} \boldsymbol{q} .
$$

All terms in equation (1) are resolved w.r.t. the body's floating frame of reference $(R)$. That's why the angular velocity of the reference frame $\omega_{R}$ have to be taken into account when the kinematic quantities velocity $\boldsymbol{v}$ and acceleration $\boldsymbol{a}_{R}$ of a particle are derived, see (Heckmann et al., 2006). The decomposition in equation (1) makes it possible to superimpose a large nonlinear overall motion of the reference frame with small elastic deformations.

The kinematic quantities are inserted into Jourdain's principle of virtual power. Subsequently, the equations of motion of an unconstrained flexible body are formulated neglecting deflection terms of higher than first order (Wallrapp, 1994, (38)):

$$
\begin{aligned}
{\left[\begin{array}{ccc}
m \boldsymbol{I}_{3} & & \text { sym. } \\
m \tilde{\boldsymbol{d}}_{C M} & \boldsymbol{J} & \\
\boldsymbol{C}_{t} & \boldsymbol{C}_{r} & \boldsymbol{M}_{e}
\end{array}\right] } & {\left[\begin{array}{c}
\boldsymbol{a}_{R} \\
\dot{\boldsymbol{\omega}}_{R} \\
\ddot{\boldsymbol{q}}
\end{array}\right]=} \\
& =\boldsymbol{h}_{\omega}-\left[\begin{array}{c}
\mathbf{0} \\
\mathbf{0} \\
\boldsymbol{K}_{e} \boldsymbol{q}+\boldsymbol{D}_{e} \dot{\boldsymbol{q}}
\end{array}\right]+\boldsymbol{h}_{e},
\end{aligned}
$$

\footnotetext{
${ }^{1}$ Both vectors and matrices are written in bold symbols, whereby vectors are of lower case letters and matrices of upper case letters.
}

where the following quantities and symbols appear:

$m$
$\boldsymbol{I}_{3}$
$\boldsymbol{d}_{C M}(\boldsymbol{q})$
$\boldsymbol{J}(\boldsymbol{q})$
$\boldsymbol{C}_{t}(\boldsymbol{q})$
$\boldsymbol{C}_{r}(\boldsymbol{q})$
$\boldsymbol{h}_{\omega}(\boldsymbol{\omega}, \boldsymbol{q}, \dot{\boldsymbol{q}})$
$\boldsymbol{h}_{e}$
$\boldsymbol{M}_{e}$
$\boldsymbol{K}_{e}$
$\boldsymbol{D}_{e}$
body mass, $3 \times 3$ identity matrix, position of center of mass, inertia tensor, inertia coupling matrix (translational), inertia coupling matrix (rotational), gyroscopic and centripetal forces, external forces, structural mass matrix, structural stiffness matrix, structural damping matrix.

In the context of the anti-roll bar modeling, the structural mass, stiffness and damping masses are gained as the result of a FE preprocessing step whose background is given in the following section. Note, that the FE preprocessing is implemented internally so that the user does not need to switch to a different modeling tool.

\subsection{FE Beam Theory}

The structural models used in multibody analysis are usually obtained from FE analysis and subsequently reduced by e.g. modal decomposition approaches. For the anti-roll bar structural models, a simple, classical finite element beam formulation is employed. Therein, the three-dimensional problem is split into a twodimensional, cross-sectional analysis and a subsequent, one-dimensional analysis along the beam's reference axis. Solving the two-dimensional problem simply involves integration of the material properties (Young's modulus, shear modulus and density) over the specified crosssectional geometry. With the resulting cross-sectional stiffness and inertia resultants, the corresponding constitutive matrix $\boldsymbol{C}$ can be built. Along with a given strain field, one arrives at the description for the force and moment distributions along the beam axis.

The one-dimensional analysis is based on (Bazoune et al., 2003), where an adjustable Timoshenko beam element was implemented, that uses linear shape functions for longitudinal displacements and torsional deformation. In order to describe the bending deformation, a cubic ansatz function is used in the lateral displacements and corresponding rotational fields. The unknown coefficients can be solved using the description for the total slopes including a constant transversal shear, the force and moment equilibrium equations and the discrete boundary conditions at both ends of the beam. These equations can then be partitioned by discerning between displacement field contribution and discrete boundary condition excitation. In a parametric space from $[0,1]$, the shape functions that are needed for the shear displacements and bending rota- 
tions read:

$$
\begin{aligned}
N_{b s}^{1} & =\frac{1}{1+\Theta}\left(1-3 \xi^{2}+2 \xi^{3}\right), \\
N_{b s}^{2} & =\frac{1}{1+\Theta}\left(3 \xi^{2}-2 \xi^{3}\right), \\
N_{b b}^{1} & =\frac{1}{1+\Theta}\left(\xi-2 \xi^{2}+\xi^{3}+1 / 2\left(2 \xi-\xi^{2}\right) \Theta\right), \\
N_{b b}^{2} & =\frac{l}{1+\Theta}\left(-\xi^{2}+\xi^{3}+1 / 2 \xi^{2} \Theta\right), \\
N_{s s}^{1} & =\frac{\Theta}{1+\Theta}(1-\xi), \\
N_{s s}^{2} & =\frac{\Theta}{1+\Theta} \xi \\
N_{s b}^{1} & =-\frac{\Theta l}{1+\Theta} 1 / 2 \xi \\
N_{s b}^{2} & =-\frac{\Theta l}{1+\Theta} 1 / 2 \xi
\end{aligned}
$$

where $\xi$ denotes the parametric coordinate along the beam axis, $l$ the beam length and $\Theta$ the bending to shear stiffness ratio. The shape function superscripts indicate the left (1) or right (2) beam end and the two-letter subscripts specify the shear (s) and bending (b) field types (first letter) and the contributing boundary condition (second letter). As previously described, their derivatives w.r.t. the parametric coordinates and the constitutive equations of cross-sectional resultants can then be used to calculate the shear and bending moment distributions along the beam and integrated using e.g. a Gauss' quadrature.

In accordance with Galerkin's weighted residual method, one can substitute into the classic expression of virtual work done by all internal forces, to arrive at a formulation for the linear element stiffness matrix $\boldsymbol{K}$, which in matrix form reads,

$$
\boldsymbol{K}=\int_{l} \boldsymbol{B}^{T} \boldsymbol{C B} \mathrm{d} l
$$

where $\boldsymbol{B}$ is the matrix of shape function derivatives describing the element strain field. Rotating all element stiffness matrices into the reference inertial coordinate system and assembly in a unified degree-of-freedom set, leads to the total stiffness matrix of the structure. In a similar fashion the consistent mass matrix $\boldsymbol{M}$ can be determined, using cross-sectional inertia resultants and the matrix of shape functions $N$. In case of the anti-roll bar a decoupled, lumped mass approach was chosen instead, where structural inertia is distributed equally at both ends of the beam. Shear center, neutral axis and center of gravity offsets from the reference line were realized as wrappers around the element stiffness and element mass matrices in order to be able to tailor the bar properties more accurately.

To facilitate a further reduction of the number of degrees of freedom, an optional Guyan's reduction (Guyan, 1964) can be performed next. The Guyan's reduction is essentially a static residualization of stiffness onto a chosen few degrees of freedom (DoF). Therein, all DoF are partitioned into an "analysis" set (index a) and an "omitted" set (index o) and a constraint w.r.t. the omitted degrees of freedom being force free is applied. The resulting transformation matrix between the analysis set and the original, full DoF set reads,

$$
\left[\begin{array}{l}
\boldsymbol{x}_{a} \\
\boldsymbol{x}_{o}
\end{array}\right]=\left[\begin{array}{c}
\boldsymbol{I} \\
-\boldsymbol{K}_{o a}^{-1} \boldsymbol{K}_{o a}
\end{array}\right]\left[\boldsymbol{x}_{a}\right] .
$$

In order for the mass and stiffness matrix to be compatible with the generalized equations of motion, a normal modes analysis is performed afterwards. The resulting eigenfrequencies and eigenvectors form a modal solution set that is used to reduce the structural degrees of freedom to the user-specified number of frequencies and mode shapes retained. Additional information required for the flexible multibody approach such as, center of gravity location, mass, inertia tensor and the inertia coupling terms are then calculated with the help of six, linearly independent rigid body mode shapes. Currently only first order inertia terms are considered, while integration of the nonlinear, second order terms is still subject to future work.

\section{Beam Based Anti-Roll Bar Model}

The introduced beam model can be utilized in various applications. In the following, we focus on a typical use case in the automotive area - the anti-roll bar.

The AntiRollBar model implemented in DLR FlexibleBodies, see the model's icon in Figure 3, allows for the modeling of flexible bars with an (almost) arbitrary geometrical shape in a user-friendly way and considers the attachments to the vehicle body and the suspensions elaborated in Section 3.1. It is tailored to be used for driving maneuvers, where frequencies higher than $20 \mathrm{~Hz}$ are out of interest but require unnecessarily large computational resources. Alternatively, the user may specify the AntiRollBar model to be employed for NVH analysis up to $400 \mathrm{~Hz}$. And last but not least, an animation of the new AntiRollBar model and its deformation field is also provided in order to assess simulation results visually.

\subsection{Anti-Roll Bar Arrangement}

To reduce the number of input parameters, the common shape of the anti-roll bar and its mounting to the vehicle's parts are considered. As can be seen in Figure 1, there are

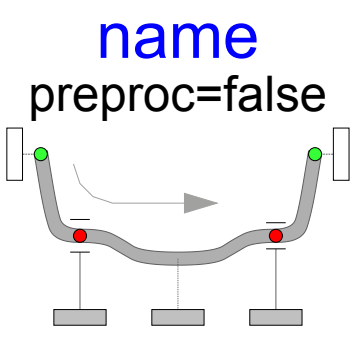

Figure 3. Icon of the new AntiRollBar model in the DLR FlexibleBodies Library. 
typically two mounts $(C)$ on the vehicle's body holding the anti-roll bar. To fulfill its operational goal of stabilizing the rolling vehicle, each of the anti-roll bar's ends is additionally connected to a suspension part on each vehicle's side $(S)$. Thus, exactly four mounting points are incorporated to connect the implemented beam based $A n$ tiRollBar. Since the common anti-roll bar is made of semifinished tube with ring-shaped cross section, the input parameters are additionally limited to outer and inner diameter of the cross section.

Considering the abovementioned restrictions, the geometry input reduces to Cartesian coordinates $x, y$ and $z$ of meaningful geometry points along the anti-roll bar's center line and its outer and inner diameters $\left(d_{o}\right.$ and $d_{i}$, respectively) at these points. Additionally, four of the points have to be marked as mountings to the vehicle. Consequently, the input reads as:

$\begin{array}{rccccc}/ / x & y & z & \text { do } & \text { di } & \text { connect } \\ 0.10 & 0.51 & 0.0 & 0.02 & 0.014 & 1 \\ -0.17 & 0.42 & 0.0 & 0.02 & 0.014 & 0 \\ -0.20 & 0.38 & 0.0 & 0.02 & 0.014 & 0 \\ \ldots & & & & & \end{array}$

Note that the four points relevant for mountings are marked in the last column by " 1 ".

The input parameters of the implemented AntiRollBar are detailed in the following sections. They reflect especially the two intended application areas of the AntiRollBar implementation - the vehicle handling analysis and the NVH. Another important aspect - which applies for both of the analyses - is the option to either input some particular predefined data of the analyzed anti-roll bar or to calculate it in preprocessing steps by the AntiRollBar model itself. The workflow of the latter is depicted in Figure 4.

\subsection{Parameters for Noise, Vibration and Harshness Analysis}

Let us consider the parameter input mask as shown in Figure 5 first. Here, the first parameter labeled Analysis specifies the option to activate the model for the NVH. Thus, the flexible body model based on a modal description will be activated in the AntiRollBar model background - in particular the ModalBody component of the DLR FlexibleBodies library. This submodel incorporates a plenty of parameters of which just three are present in the input mask of the AntiRollBar, namely:

- fileFlexBody - a shared name of files which describe the flexible body dynamics and animation - SID ${ }^{2}$ and $o b j{ }^{3}$, respectively. To simplify the input, this name is required without the file suffix, assuming that both SID and obj files of the modeled anti-roll bar have the same name.

\footnotetext{
${ }^{2}$ Standard Input Data file

${ }^{3}$ File with $3 \mathrm{D}$ data in Wavefront OBJ format, see e.g http://www.fileformat.info/format/wavefrontobj/egff.htm
}

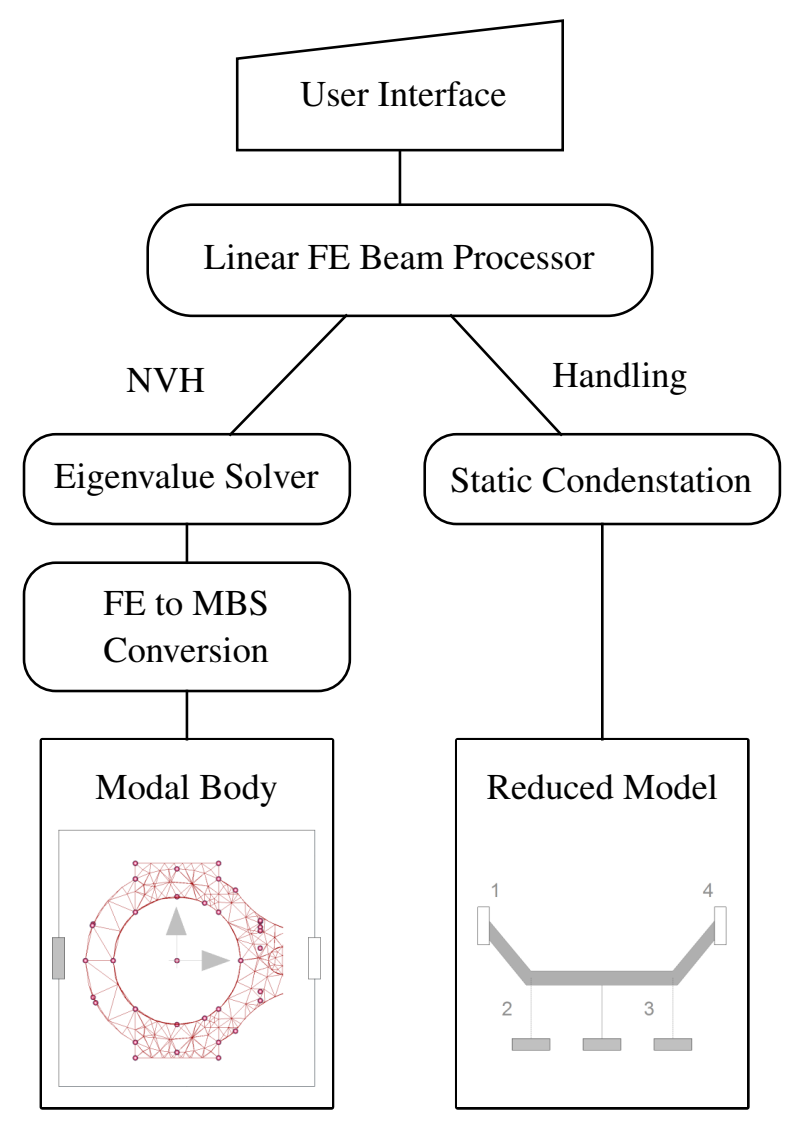

Figure 4. Preprocessing steps of the AntiRollBar model with two branches for NVH or vehicle handling scenarios.

- $n \_$modes - the number of eigenmodes to be considered.

- Nodes - (exactly four) specific node numbers to be associated to the AntiRollBar connector frames.

At this point, the meaning of the parameter preprocessing shall be further explained. In Figure 5, this parameter is set to false. Thus, it is required that the user inputs both the SID and obj files by defining fileFlexBody and the indexes of the connector nodes using Nodes. The information on the number of eigenmodes is, in contrast, not relevant, and therefore disabled.

The situation changes when the user wishes to generate the data in a preprocessing step setting preprocessing = true. Then, fileFlexBody indicates no more the name of the existing files but the name of files to be generated by the preprocessor, see below, and $n \_$modes is the information being additionally required. The parameter array Nodes is then read from the last column of the geometry table shown in Section 3.1. This table, called geometry, has to be saved in the input file indicated by the parameter fileName. This file must additionally contain a table called material with anti-roll bar material properties. In particular, material density $\rho$, Young's modulus $E$ and shear modulus $G$ are required in the current AntiRollBar implementation. An example on the input data file format is given in Appendix A. For better understanding on the 


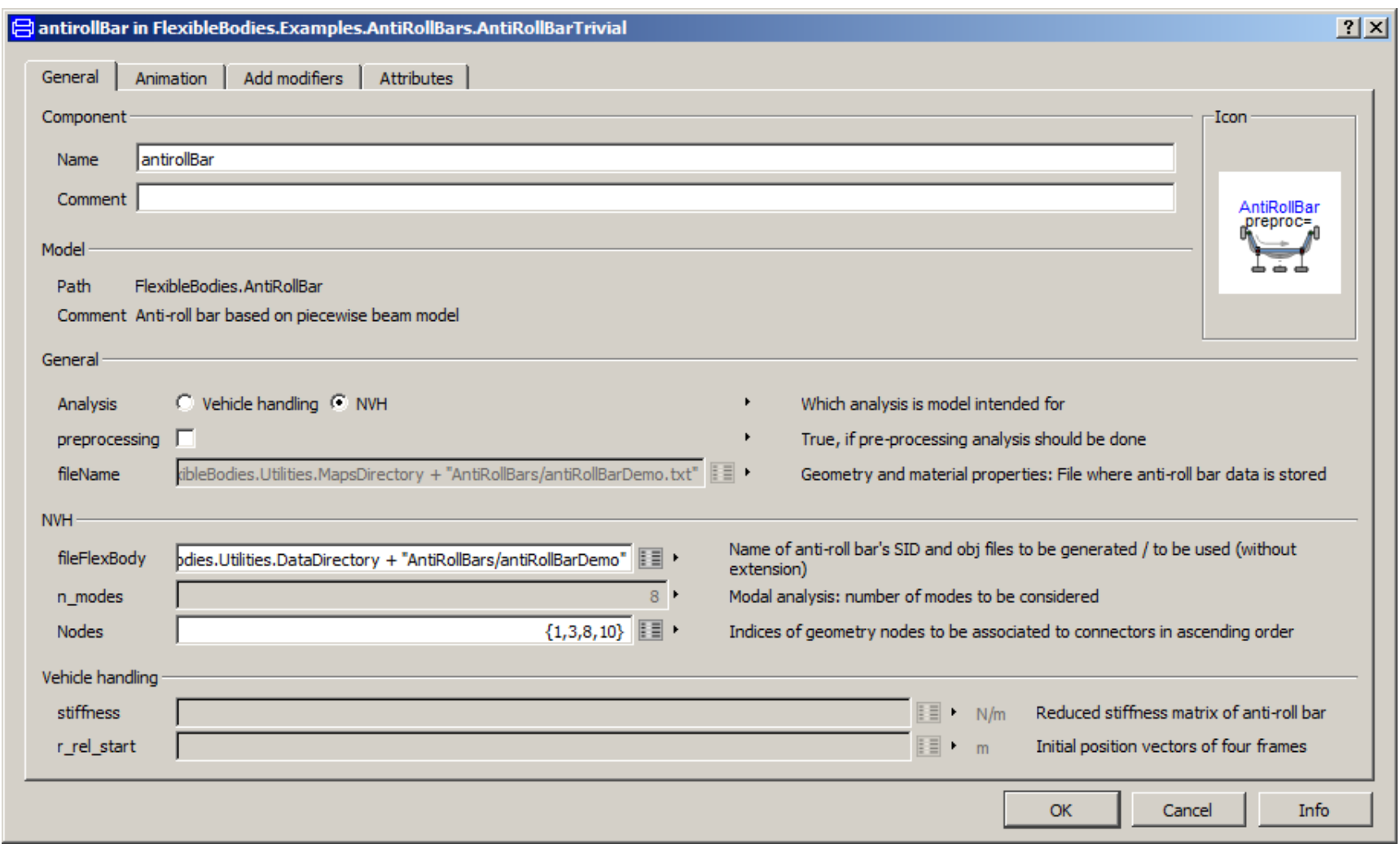

Figure 5. Parameter menu to specify AntiRollBar model.

preprocessing procedure, the steps are highlighted in the left branch of Figure 4.

As mentioned above, there is a particular output if the preprocessing is enabled for the NVH analysis. Then, two files are generated - one in SID and the other one in wavefront format. The SID file, see (Wallrapp, 1994), contains the modal reduced anti-roll bar structure where the input $n \_$modes defines the number of retained modes. The Wavefront file (signalized by an obj suffix) enables the visualization of the anti-roll bar, see Figure 6. As common to DLR FlexibleBodies library, the anti-roll bar is visualized by both solid and wireframe elements, whereby the number of vertices and face elements of the wireframe grid can be influenced by the user.

\subsection{Parameters for Vehicle Dynamics}

The next Analysis option is the one for the vehicle dynamics. This case utilizes the following parameters:

- stiffness - a $4 \times 4$ stiffness matrix and

- r_rel_start - a $4 \times 3$ matrix containing Cartesian coordinates to get the proper position of the four connector frames.

The simplified matrix stiffness applies according to equation (5) for purely vertical DoF's considered in the four anti-roll bar mountings. If both stiffness and $r \_r e l \_s t a r t$ should be generated by preprocessor, the input simplifies to only fileName. The right branch of Figure 4 shows all necessary preprocessing steps in this case.

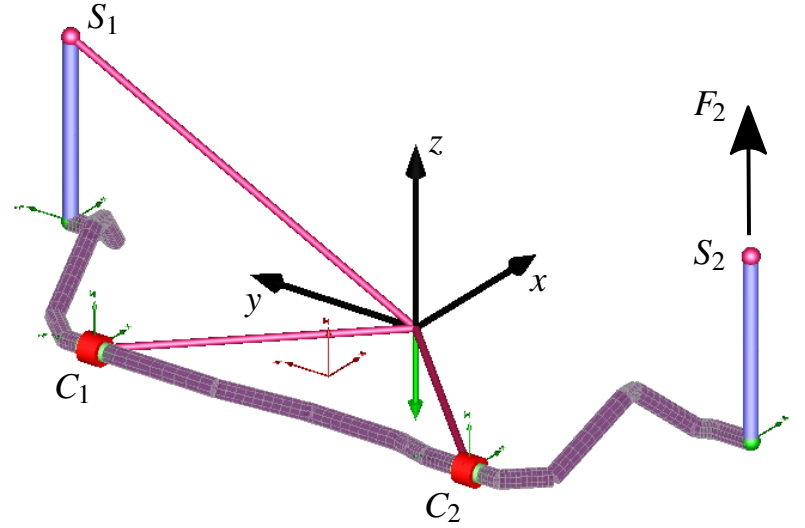

Figure 6. Anti-roll bar model with a connecting link on each side.

\section{Simulation Experiment}

For parametrization of the AntiRollBar, an important question concerns the number of eigenmodes which have to be considered when generating the SID file for NVH analysis. This influences not only the simulation results significantly, but also the simulation time.

To evaluate this phenomena we have defined a simple virtual experiment, depicted in Figure 6, which is intended to excite all the considered eigenmodes - similarly to an experimental modal analysis with single point excitation. In this experiment, the anti-roll bar is connected to the inertial frame at the vehicle's body mounts $C_{1}$ and $C_{2}$ via two 
Table 1. Eigenfrequencies of the evaluated anti-roll bar.

\begin{tabular}{cc}
\hline Eigenmode & Eigenfrequency / Hz \\
\hline 1 & 61,7 \\
2 & 100,7 \\
3 & 121,9 \\
4 & 139,9 \\
5 & 217,2 \\
6 & 321,4 \\
7 & 375,0 \\
8 & 467,9 \\
9 & 521,6 \\
10 & 556,8 \\
11 & 640,0 \\
12 & 817,8 \\
13 & 927,0 \\
\hline
\end{tabular}

spring-damper elements. Additionally, a link with two ball joints - a typical connection in automotive applications is used to join one anti-roll bar's end to the inertial frame at fixed point $S_{1}$. The other end is connected similarly to point $S_{2}$ which, in contrast, can freely move in vertical direction ( $z$-axis in Figure 6).

In the simulation scenario, the anti-roll bar is first preloaded at $S_{2}$ with a constant vertical force $F_{2}$. After some time period which guarantees that the mechanism is at rest, the link connection is "released" by fast drop of the force towards zero. Thus, a desired damped oscillation of the anti-roll bar around its unloaded state is induced. With this procedure, two criteria can be assessed: a) the overall stiffness of the anti-roll bar by relating the applied force $F_{2}$ to the static displacement of point $S_{2}$ and b) the simulation time $t_{\mathrm{CPU}}$.

The anti-roll bar used in this example is asymmetric in vehicle's longitudinal plane, see also Appendix A for particular data input. For this geometry, the eigenmodes up to the frequency $f=1000 \mathrm{~Hz}$ are given in Table 1 .

The Figures 7 and 8 show the resulting overall stiffness $c_{\text {all }}$ of the anti-roll bar and the simulation times $t_{\mathrm{CPU}}$, respectively, over the number of considered modes $n \_$modes. Since there is a significant drop in $c_{\text {all }}$ between $n \_$modes $=1$ and $n \_$modes $=2$ in Figure 7 a), the parameter $c_{\text {all }}$ is plotted in Figure $7 \mathrm{~b}$ ) without the value for $n \_$modes $=1$.

The deployment $c_{\text {all }}$ in Figure $7 \mathrm{~b}$ ) reflects the influence of single modes on the anti-roll bar stiffness. The eigenmode 2 is the most significant as there is an extraordinary change in the stiffness. Another change can be observed for eigenmode 6 . For higher eigenmodes, the modifications in $c_{\text {all }}$ are marginal and - as can be seen in Figure 8 only lead to unnecessary increase of the simulation time.

Consequently, at least the first six eigenmodes, i.e. $n \_$modes $=6$, should always be considered for the evaluated anti-roll bar's geometry. This applies even for NVH analyses in lower frequency range of interest. A higher
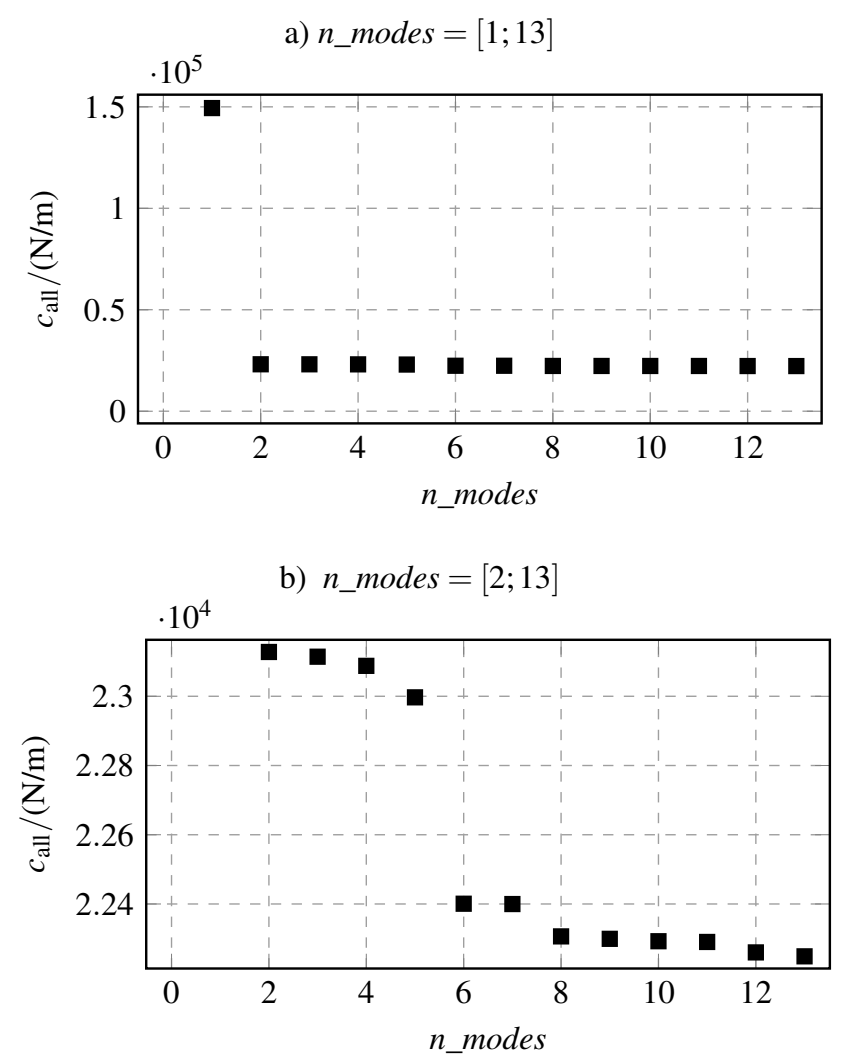

Figure 7. Anti-roll bar stiffness $c_{\text {all }}$ for changing number of modes.

number of eigenmodes could nevertheless be introduced, but then a progressive increase of simulation time has to be taken into account.

\section{Conclusions}

The presented automotive anti-roll bar model can be applied for both the vehicle dynamics and the NVH analysis. The simulation experiment emphasizes the changes in model behavior depending on the structure's eigenmodes and shows how to indicate significant eigenmodes, which should always be included in the analyses. Due to the dependency of the eigenmodes on the anti-roll bar's geom-

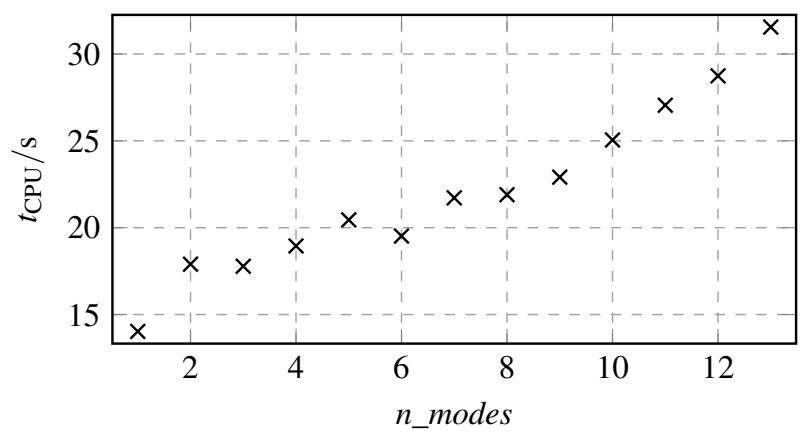

Figure 8. Simulation time $t_{\mathrm{CPU}}$ for $n \_$modes $=[1 ; 13]$. 
etry and material data, this identification has to be performed for each particular anti-roll bar.

The future development of the presented model will focus on the incorporation of structural damping as input parameter. Moreover, higher order models should be implemented for higher model fidelity.

\section{Acknowledgements}

The authors would like to thank to Frank Su and Jim Alanoly from Ford Motor Company, Dr. Bilal Maiteh from Dassault Systemes and John Batteh, John Griffin and Johan Andreasson, all from Modelon AB, for initialization of this work and important support provided at the beginning period of the project.

\section{References}

A. Bazoune, Y. A. Khulief, and Stephen N. G. Shape Functions of Three-Dimensional Timoshenko Beam Elements. Journal of Sound and Vibration, pages 473-480, 2003.

R. J. Guyan. Reduction of stiffness and mass matrices. AIAA Journal, 3:380, 1964.

A. Heckmann, M. Otter, S. Dietz, and J. D. López. The DLR FlexibleBody library to model large motions of beams and of flexible bodies exported from finite element programs. In 5th International Modelica Conference, pages 85-95, 2006.

B. Heißing and M. Ersoy. Chassis Handbook. Vieweg+Teubner Verlag, 1 edition, 2011. DOI 10.1007/978-3-8348-9789-3.

G. Rill. Road Vehicle Dynamics: Fundamentals and Modeling. CRC Press, 2012. ISBN 978-1-4398-3898-3.

O. Wallrapp. Standardization of flexible body modeling in multibody system codes, Part 1: Definition of standard input data. Mechanics of Structures and Machines, 22(3):283-304, 1994.

\section{A Appendix: Example of Input Data File}

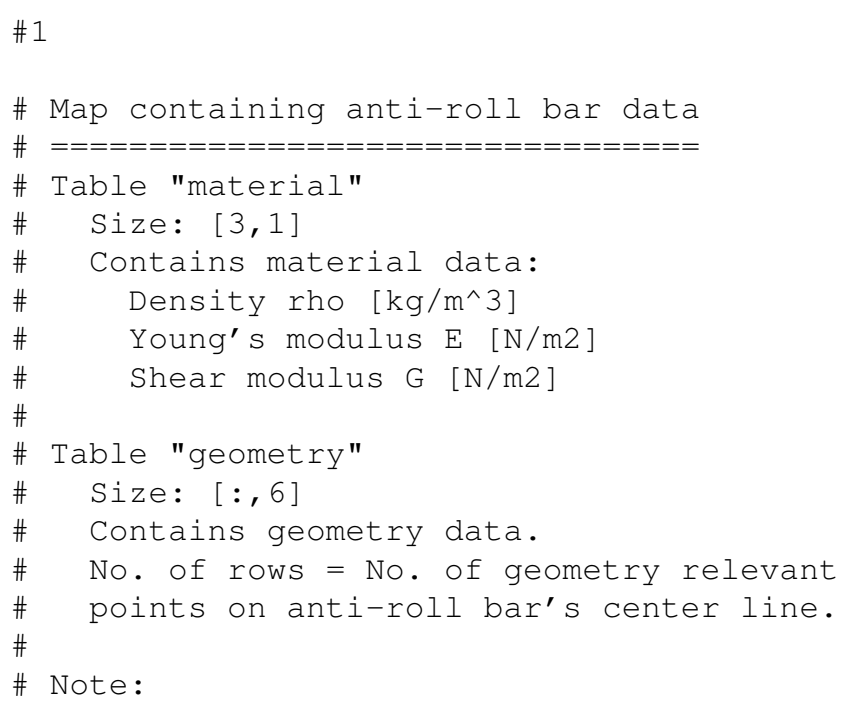

$\# 1$

1) Both symmetric and non symmetric anti-roll bar is applicable.

2) Exactly four connectors are available at the moment, i.e. the last column must contain exactly four non zero integers (the value of it plays no role).

3) The points are given successively from the left end-point to the right end-point.

4) Diameter outer must always be greater then diameter inner.

double material $(3,1)$

$7.86 \mathrm{e} 3$

$2.07 e 11$

$7.90 e 10$

\# \# $x$ y $z$ do di connect double geometry $(21,6)$

$\begin{array}{llllll}0.06 & 0.62 & 0.03 & 0.024 & 0.018 & 1\end{array}$

$\begin{array}{llllll}0.06 & 0.60 & 0.03 & 0.024 & 0.018 & 0\end{array}$

$\begin{array}{llllll}0.04 & 0.60 & 0.08 & 0.024 & 0.018 & 0\end{array}$

$\begin{array}{llllll}0.00 & 0.60 & 0.10 & 0.024 & 0.018 & 0\end{array}$

$\begin{array}{llllll}-0.06 & 0.58 & 0.05 & 0.024 & 0.018 & 0\end{array}$

$\begin{array}{lllllll}-0.11 & 0.56 & 0.04 & 0.024 & 0.018 & 0\end{array}$

$\begin{array}{lllllll}-0.16 & 0.51 & 0.04 & 0.024 & 0.018 & 0\end{array}$

$\begin{array}{llllll}-0.16 & 0.46 & 0.04 & 0.024 & 0.018 & 1\end{array}$

$\begin{array}{lllllll}-0.16 & 0.10 & 0.04 & 0.024 & 0.018 & 0\end{array}$

$\begin{array}{llllll}-0.23 & -0.06 & 0.02 & 0.024 & 0.018 & 0\end{array}$

$\begin{array}{llllll}-0.23 & -0.24 & 0.02 & 0.024 & 0.018 & 0\end{array}$

$\begin{array}{llllll}-0.23 & -0.30 & 0.02 & 0.024 & 0.018 & 0\end{array}$

$\begin{array}{llllll}-0.16 & -0.41 & 0.04 & 0.024 & 0.018 & 0\end{array}$

$\begin{array}{llllll}-0.16 & -0.46 & 0.04 & 0.024 & 0.018 & 1\end{array}$

$\begin{array}{lllllll}-0.16 & -0.51 & 0.04 & 0.024 & 0.018 & 0\end{array}$

$\begin{array}{lllllll}-0.11 & -0.56 & 0.04 & 0.024 & 0.018 & 0\end{array}$

$\begin{array}{llllll}-0.06 & -0.58 & 0.07 & 0.024 & 0.018 & 0\end{array}$

$\begin{array}{llllll}0.02 & -0.60 & 0.10 & 0.024 & 0.018 & 0\end{array}$

$\begin{array}{llllll}0.05 & -0.60 & 0.08 & 0.024 & 0.018 & 0\end{array}$

$\begin{array}{llllll}0.06 & -0.60 & 0.03 & 0.024 & 0.018 & 0\end{array}$

$\begin{array}{llllll}0.06 & -0.62 & 0.03 & 0.024 & 0.018 & 1\end{array}$ 
